\title{
Active structural elements within a general vibration control framework
}

Jan Holterman Theo J.A. de Vries

\author{
presented at the \\ $1^{\text {st }}$ IFAC Conference on Mechatronic Systems \\ Mechatronics 2000 \\ Darmstadt, Germany, 18-20 September 2000
}

published in

R. Isermann (editor), (2000), Proc. $1^{\text {st }}$ IFAC Conference on Mechatronic Systems

'Mechatronics 2000', Darmstadt, Germany, pp. 997-1002 


\title{
ACTIVE STRUCTURAL ELEMENTS WITHIN A GENERAL VIBRATION CONTROL FRAMEWORK
}

\author{
Jan Holterman Theo J.A. de Vries \\ Cornelis J. Drebbel Institute for Systems Engineering, EL-RT, University of Twente \\ PO Box 217, 7500 AE Enschede, The Netherlands \\ Phone: +31 53489 2788, Fax: +31534892223 \\ mechatronics@rt.el.utwente.nl,http://www.rt.el.utwente.nl/smartdisc
}

\begin{abstract}
High-precision machines typically suffer from small but annoying vibrations. As the most appropriate solution to a particular vibration problem is not always obvious, it may be convenient to cast the problem in a more general framework. This framework may then be used for frequency response analysis, which, together with close examination of the disturbance sources, leads to a solution in general structural terms, like 'vibration isolation', 'stiffness enhancement' or 'damping augmentation'. In case it is not possible or unpractical to control the vibration passively, a solution based on active structural elements may be considered.
\end{abstract}

Keywords: active control, active elements, frequency response methods, modal control, mode analysis, structural parameters, systems engineering, vibration dampers

\section{INTRODUCTION}

High-precision machines typically suffer from small but annoying vibrations. These may either be induced by environmental vibrations transmitted by the floor or the surrounding air, or result as an unwanted side effect due to actuator activity inside the machine. A straightforward solution to many vibration problems is to increase the stiffness within a machine. There is however a practical limit in passively increasing the stiffness of a structure. Furthermore, due to dedicated structural design rules (Koster, 1998), vibrations in high-precision machines are typically badly damped (Van Schothorst, 1999). Introducing additional passive damping into high-precision structures however is complicated, as the stresses and strains to be damped are very small (Fanson, et al., 1990).

Because of the indicated passive restrictions, worldwide much research effort is put into active vibration control strategies. Because of the relative ease of implementation and stability robustness, especially active structural elements for vibration control with co-located sensing and actuation, have gained much interest (Bronowicki, et al., 1994; Anderson, et al., 1997; Preumont, 1997). ACX ${ }^{1}$ for example has developed the SmartPack ${ }^{\mathrm{TM}}$ (Spangler, et al., 1997); $\mathrm{JPL}^{2}$ has developed an active strut (Anderson, et al., 1990). At the Cornelis J. Drebbel Institute for Systems Engineering at the University of Twente, research is devoted to the development of a Smart Disc, which is envisioned as a load-bearing active structural element, with integrated sensing, actuation and control (Holterman, et al., 1998; Van Schothorst, 1999).

\footnotetext{
${ }^{1}$ Active Control eXperts, Inc., 215 First Street, Cambridge, MA 02142

${ }^{2}$ Jet Propulsion Laboratory, California Institute of Technology, 4800 Oak Grove Drive, Pasadena, CA 91109-8001
} 
The past decades, much research has been aimed at the solution of several dedicated problems in the area of vibration control. The distinctive aim of the present paper is to provide a framework for classifying generalized vibration problems in highprecision machines, and to indicate the virtues of active structural elements within this framework. As such, the central questions addressed here are:

\section{Given a certain generalized vibration problem.}

\section{How to control it in general structural terms?}

2. How to control it using active structural elements?

\section{MODELLING A HIGH-PRECISION MACHINE}

In this paper a largely stylized model of a highprecision machine is considered (see figure 1). The model is kept as general, and thus as simple, as possible. As a consequence, the model represents only one single dominant vibration mode of the machine itself, caused by the fact that the connection $\left(k_{2}\right)$ between two important parts of the machine is not infinitely stiff. Relative movement of these parts, referred to as the upper frame $\left(m_{2}\right)$ and the base frame ( $m_{1}$; with, in general, $\left.m_{1}>m_{2}\right)$, is undesirable, as this is assumed to preclude proper operation of the machine.

In order to minimize the effect of inevitable floor vibrations $x_{\mathrm{d}}(t)$, high-precision machines are often resiliently supported (indicated by a stiffness $k_{1}$ parallel to a damper $d_{1}$; with, of course, $k_{1}<<k_{2}$ ). As a consequence, the model incorporates a second vibration mode which is assumed to correspond roughly to the joint movement of the upper frame and the base frame with respect to the floor.

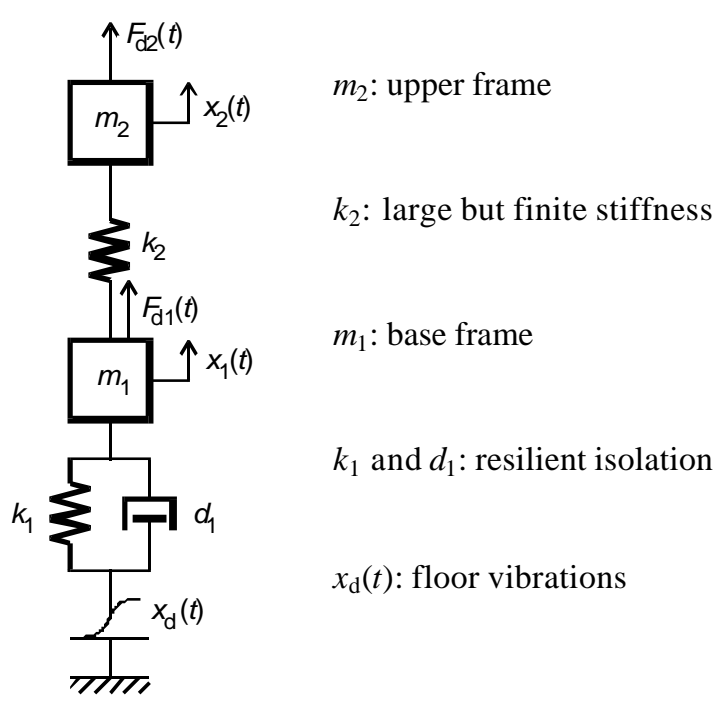

Fig. 1. Stylized model of a high-precision machine
Besides floor vibrations, in practice actuators within the machine and acoustic waves guided by the surrounding air may also excite vibrations. This implies that in the model, at least two other disturbance sources should be incorporated: disturbing forces on both masses, $F_{\mathrm{d} 1}(t)$ and $F_{\mathrm{d} 2}(t)$.

It is important to note that the model presented here is truly a drastic simplification of a high-precision machine in practice. The model for instance displays only one dimension, whereas reality is threedimensional. Nevertheless, in order to come up with a general as possible framework for 'high-precision machine vibration control' the model should be preferably as simple as possible, incorporating only the phenomena that are of most importance for understanding the nature of the vibration problem. Damping between the upper frame and the base frame for instance is not modelled; its magnitude, and therefore its influence, is assumed to be rather small.

\section{FREQUENCY RESPONSE ANALYSIS}

For proper operation of the modelled machine, the positional difference $x_{2}-x_{1}$ should be as small as possible, despite the fact that two disturbing forces and a disturbing movement act upon the structure. In order to come up with means to minimize the positional difference, its response to the three disturbance sources should be examined (for simplicity, the damper $d_{1}$ is left out of the analysis):

$$
x_{2}-x_{1}=-\frac{m_{2} s^{2}}{D_{0}(s)} F_{d 1}+\frac{m_{1} s^{2}+k_{1}}{D_{0}(s)} F_{d 2}-k_{1} \frac{m_{2} s^{2}}{D_{0}(s)} x_{d}
$$

with

$$
D_{0}(s)=m_{1} m_{2} s^{4}+\left(k_{1} m_{2}+k_{2} m_{2}+k_{2} m_{1}\right) s^{2}+k_{1} k_{2} .
$$

The frequency responses of the positional difference to the three disturbance sources (see figure 2) are characterized by three frequencies, for $k_{1}<<k_{2}$ and $m_{1}>m_{2}$ given by:

resonances:

$$
\omega_{e 1}^{2} \approx \frac{k_{1}}{m_{1}+m_{2}}, \omega_{e 2}^{2} \approx k_{2} \frac{m_{1}+m_{2}}{m_{1} m_{2}},
$$

antiresonance:

$$
\omega_{a}^{2}=\frac{k_{1}}{m_{1}}
$$

The generalized model of figure 1 is easily seen to be characterized by six structural parameters: two damping values, determining the height of the 


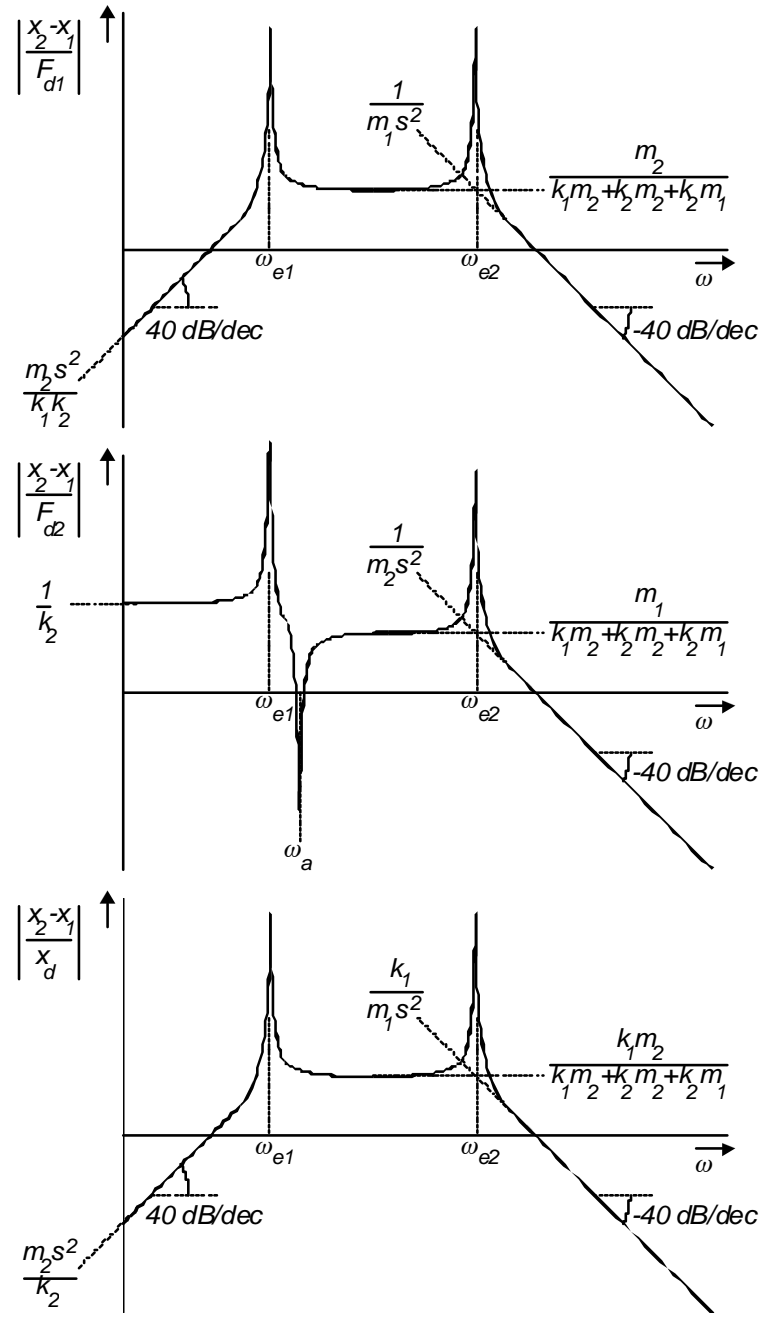

Fig. 2. Frequency responses from disturbances to positional difference

resonance peaks, and two masses and two stiffness values which together determine the asymptotes of the responses. With respect to the latter four structural parameters, their influence on the frequency responses is summarized in figure 3 .

It is important to note that the responses shown in figure 2 and 3 only represent a view on half of the problem. The other half of the problem relates to the dominant disturbance sources. Vibration control is in general only possible after all aspects of the vibration problem at hand have been examined thoroughly, i.e., aspects concerning the structure suffering from vibration as well as aspects concerning the disturbance sources (Mead, 1998).

\section{VIBRATION CONTROL IN GENERAL STRUCTURAL TERMS}

In general, the first attempt to control the vibration should be in reduction of the disturbance from the source (Mead, 1998). If this is not possible or unpractical, the only solution left is in reshaping the responses, that is, in lowering the resonance peaks and/or manipulating the response asymptotes. Once the desired changes in the responses have been established, the solution to the problem can easily be formulated in terms of the structural parameters appearing in the model.

Addition of damping, which corresponds to lowering a resonance peak, for instance is easily seen to be a successful approach only in case the vibration problem is caused predominantly by an initially badly damped structural resonance. The overall response shape however is hardly affected by 'damping augmentation'. This implies that, in case the vibration problem manifests itself in a rather broad frequency band, sole damping augmentation is in general not useful. With respect to figure 2 , in that case the responses should be lowered in a certain non-resonant frequency range, which can only be achieved by a change of the general structural parameters mass and/or stiffness, not damping (Mead, 1998).

In finding the most appropriate means to reshape the frequency responses, figure 3 can be used in a very convenient way. In case the main disturbance source is the floor vibration $x_{\mathrm{d}}$, vibration isolation (response sets $c$ and $d$ ) is usually considered. Comparison of both response sets now indicates that in general an increase of the base frame mass $m_{1}$ is preferred above a decrease of the support stiffness $k_{1}$, the main difference being in the responses to $F_{\mathrm{d} 1}(t)$.

Likewise, response sets $a$ and $b$ indicate that an increase of the machine stiffness $k_{2}$ (stiffness enhancement) is preferable above a decrease of the mass $m_{2}$ of the upper frame, the main difference here being in the responses to $F_{\mathrm{d} 2}(t)$. Note that, intuitively, an increase of the stiffness $k_{2}$ can also be seen to be the overall best solution

\section{ACTIVE VIBRATION CONTROL}

Once the solution to the vibration problem has been formulated in general terms, it should be determined how to implement the solution in practice. It was already mentioned in the introduction that for highprecision machine vibration problems, implementation by passive means might be impossible or unpractical. In that case a solution based on active elements may be considered (Preumont, 1997; Mead, 1998).

In this paper we consider two slightly distinct active vibration control approaches, both based on colocated sensing and actuation. The virtues of both approaches are discussed for the simplest possible model, describing only a single mode of a structure. We consider a mass-spring system at a vibrating floor, $x_{\mathrm{d}}(t)$ (figure 4). A position actuator $u(t)$ and a sensor, both appropriately co-located, together with a 


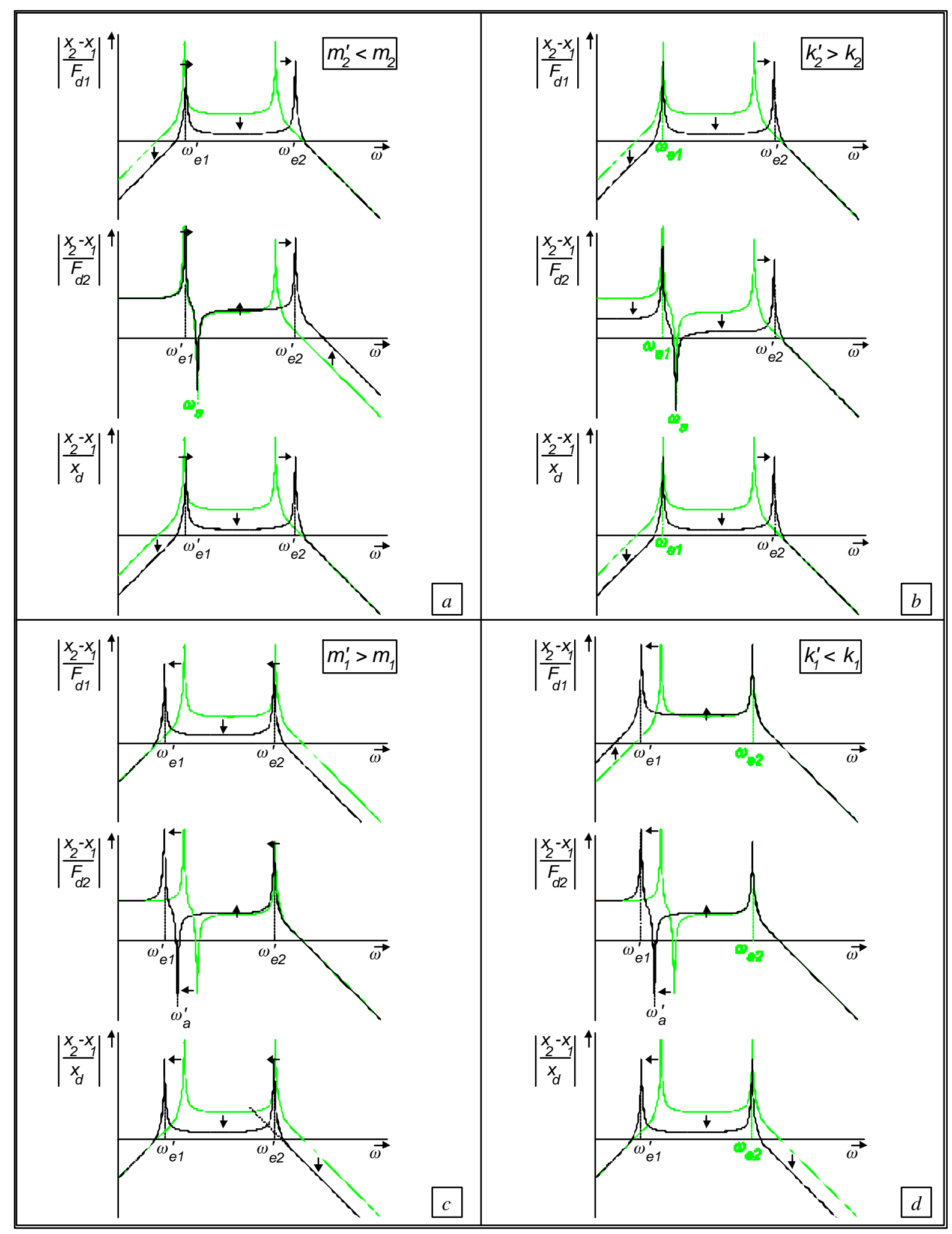

Fig. 3. Overview of effects of parameter changes on frequency responses 

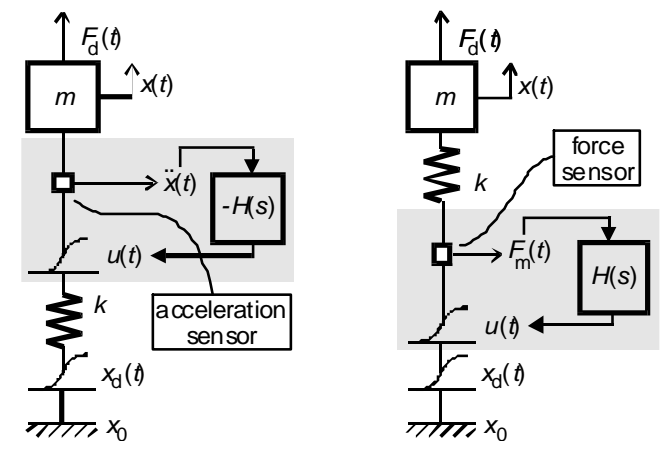

Fig. 4. Acceleration feedback (left) and force feedback (right)

controller constitute the active structural element (indicated by the shaded boxes in figure 4) the Drebbel Institute aims for.

\subsection{Acceleration feedback}

First we consider the actuator to be steered upon a measurement of the acceleration of the mass. Application of a static feedback law:

$$
u=-H(s) s^{2} x=-H_{0} s^{2} x
$$

boils down to a virtual mass change, according to

$$
m^{\prime}=m+H_{0} k
$$

In case the feedback law is dynamic rather than static, it is also possible to add damping to the system: Direct Velocity Feedback (Preumont, 1997) is known to correspond to a damper between ' $x_{0}$ ' and the mass.

\subsection{Force feedback}

Next we consider the actuator to be steered upon force measurement in the supporting structure. Application of a static feedback law:

$$
u=H(s) F_{m}=H_{0} F_{m}
$$

then boils down to a virtual stiffness change, according to:

$$
k^{\prime}=k /\left(1+k H_{0}\right)
$$

In case the feedback law is dynamic rather than static, it is also possible to add damping to the system: Integral Force Feedback (Preumont, 1997) is known to correspond to a damper between the mass and the vibrating floor $\left(x_{\mathrm{d}}\right)$.
Table 1 Effect (in terms of figure 3 ) of active element insertion in the machine model (figure 1)

\begin{tabular}{ccc}
\hline $\begin{array}{c}\text { Active structural } \\
\text { element location }\end{array}$ & $\begin{array}{c}\text { Acceleration } \\
\text { feedback }\end{array}$ & $\begin{array}{c}\text { Force } \\
\text { feedback }\end{array}$ \\
\hline between $m_{2}$ and $k_{2}$ & $a$ & $b$ \\
between $k_{2}$ and $m_{1}$ & - & $b$ \\
between $m_{1}$ and $k_{1}$ & $c$ & $d$ \\
between $k_{1}$ and $x_{\mathrm{d}}$ & - & $d$ \\
\hline
\end{tabular}

The effect of the proposed active structural elements in terms of the response sets shown in figure 3, (termed $a, b, c$, or $d$ ) is summarized in table 1 .

An important remark that should be made here with respect to active vibration control is the fact that it is only effective within a limited bandwidth. In general a vibration control system would consist of an active part for low frequencies, and a passive part for higher frequencies (Hansen, and Snyder, 1997).

\section{EXAMPLE: 'SMART DISC PROOF-OF- CONCEPT' EXPERIMENT}

As an example of the use of the framework presented in this paper, we consider the 'Smart Disc proof-ofconcept' experiment, in which the deflection of a simple beam was compensated for actively (Holterman, et al., 1998). The experiment was set up such that a disturbing force could be applied at the top of the beam. This force, being far more dominant than any other disturbance source, was intended to act in a limited frequency range $(0.01-10 \mathrm{~Hz})$, far below the lowest natural frequency of the system.

The experiment as described above, can easily be cast in the general framework presented in this paper. It is obvious that we should only be concerned about the response to $F_{\mathrm{d} 2}$. The frequency range of interest is below $\omega_{e 2}$, implying that a resonance is not the problem; damping augmentation thus is not useful. From figure 3 it is furthermore easily seen that the structural parameter to be changed is $k_{2}$. We thus conclude to consider solution $b$.

As our intention has been to evaluate the concept of vibration control using an active structural element, we refer table 1, and decide upon force feedback and insertion between $m_{1}$ and $k_{2}$ (due to the experiment setup insertion between $k_{2}$ and $m_{2}$ was not possible). The experiment performed showed that the prototype Smart Disc was able to suppress vibration in the intended frequency range 10 to $30 \mathrm{~dB}$, corresponding to an active stiffness enhancement factor of 3 to 30 (figure 5). 


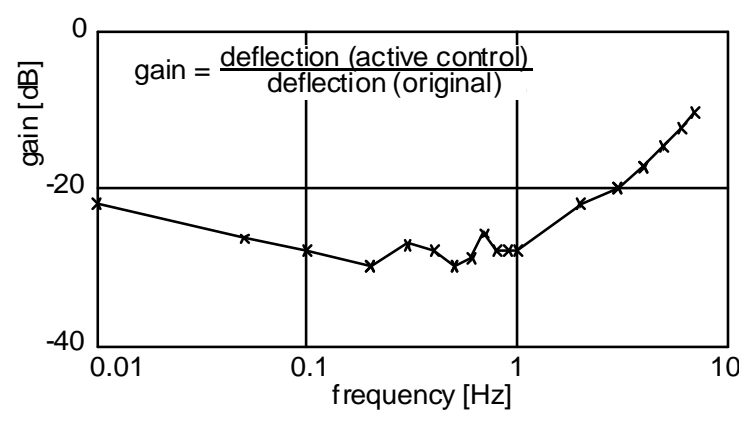

Fig. 5. Experiment frequency response

Subsequent experiments with a block-shaped disturbing force revealed that a further increase of the control bandwidth should be used for an increase of the stiffness in a broader frequency range, rather than the addition of damping at the resonance frequency of the system (figure 6).

\section{CONCLUSION}

With respect to high-precision machine vibration problems, the following general method for arriving at a practical solution can be used.

(1) Formulate a competent model, incorporating the dominant harmful vibration modes of the system at hand and the dominant disturbance sources, and characterize the disturbance sources in the frequency domain.

(2) If the problem is dominated by a resonance, then try to increase the damping (by passive means); otherwise, based on figure 3 , reshape the frequency responses by changing the general structural parameters mass and/or stiffness (passively).

(3) In case it turns out impossible or unpractical to implement the proposed solution in general terms by passive means, consider a solution based on active structural elements, based on figure 3 and table 1 .

\section{REFERENCES}

Anderson, E.H., D.M. Moore, J.L. Fanson, and M.A. Ealey (1990). Development of an active truss element for control of active structures. In: Optical Engineering, Vol. 29, No. 11, pp. 13331341.

Anderson, E.H., M.D. Holcomb, A.X. Bogue, and F.R. Russo (1997). Integrated Electro-mechanical Devices for Active Control of Vibration and

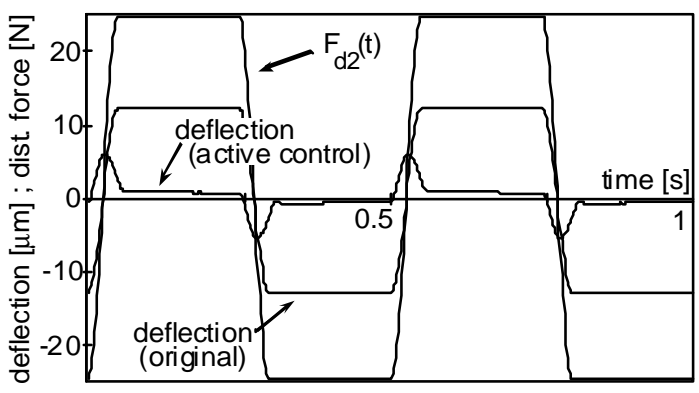

Fig. 6. Response to block-shaped disturbance

Sound. Presented at Adaptive Structures and Materials Systems Symposium, International Mechanical Engineering Congress and Exposition, Dallas, TX, November 1997.

Bronowicki, A.J. J. Innis, S. Casteel, G. Dvorsky, O. Alvarez, and E. Rohleen (1994). Active Vibration Suppression using Modular Elements. In: Proc. SPIE Smart Structures and Intelligent Syetems, Vol. 2190, pp. 717-728

Fanson, J.L., E.H. Anderson, and D. Rapp (1990). Active structures for use in precision control of large optical systems. In: Optical Engineering, Vol. 29, No. 11, pp. 1320-1327.

Hansen, C.H., and S.D. Snyder (1997). Active Control of Noise and Vibration, Chapman \& Hall, London, Great Britain.

Holterman, J., T.J.A. de Vries, and M.P. Koster (1998). Experiment to evaluate the feasibility of the Smart Disc concept. In: Proc. $6^{\text {th }} U K$ Mechatronics Forum International Conference Mechatronics '98, Skövde, Sweden, pp. 217-222.

Koster, M.P. (1998). Constuctieprincipes (in Dutch), Twente University Press, Enschede, The Netherlands.

Mead, D.J.(1998). Passive Vibration Control, Wiley \& Sons, Chichester, Great Britain.

Preumont, A. (1997). Vibration Control of Active Structures, An Introduction, Kluwer Academic Publishers, Dordrecht, The Netherlands.

Spangler, R.L., F.M. Russo, and D.A. Palombo (1997). A Compact Integrated Piezoelectric Vibration Control Package. In Smart Structures and Integrated Systems, Proc. SPIE Smart Structures and Materials, Vol. 3041, pp. 728-740.

Van Schothorst, G. (1999). Active Vibration Control using Piezoelectric Smart Discs. In: Mathematics and Control in Smart Structures, Proc. SPIE Smart Structures and Materials, Vol. 3667, pp. 637-648. 